\title{
Effect of Interfacial Tension on Absorption of toluene / butyl acetate
}

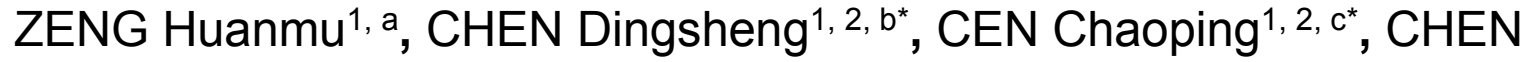 \\ Zhihang $^{1,2}$, FANG Ping ${ }^{1,2}$, ZHANG Yukui ${ }^{1}$, CHEN Xiongbo ${ }^{1}$, and TANG Zijun ${ }^{1}$ \\ ${ }^{1}$ South China Institute of Environmental Sciences, Ministry of Environmental Protection, Guangzhou \\ 510655, China \\ 2 The Key Laboratory of Water and Air Pollution Control of Guangdong Province, Guangzhou \\ 510655, China \\ a zenghuanmu@scies.org, b chendingsheng@scies.org, ' cenchaoping@scies.org
}

Keywords: Interfacial Tension, toluene, absorption

Abstract. Surface tension/interfacial tension plays an important role in the process of VOCs absorption. A series of experiments were conducted to investigate the relationship between the interfacial tension and absorption efficiency of toluene. The results show that the performance of SCOP absorbent has the lowest surface tension and the best absorption. The absorption mechanism is that, the addition of additives dissolved in water to prepare composite absorbent, which cloud reduce the interfacial tension between absorbed gas and absorbent, and enhance the ability of gas-phase pollutants dissolved in the liquid phase, therefore improve the absorption efficiency of toluene.

\section{Introduction}

With the rapid development of urbanization and industrialization in China, the VOCs emissions increase rapidly ${ }^{[1-2]}$. Industrial activity is an important source of VOCs, involving a wide range of industries, a wide range of pollutants, and a complex composition. Common species are hydrocarbons (alkanes, alkenes and aromatics), ketones, esters, alcohols, phenols, aldehydes, amines, nitriles and other organic compounds, of which the largest industrial emissions of substances for the three Benzene (benzene, toluene, xylene) and halogenated hydrocarbons, benzene and halogenated hydrocarbons are also highly toxic substances ${ }^{[3]}$. Removal of volatile organic compounds (VOCs) has attracted much attention recently because VOCs have caused environmental and health problems, such as urban smog, destruction of ozone layer, infection to eyes, nose and even cancer ${ }^{[4]}$.

In the organic waste gas treatment technology, the liquid absorption method is an economically feasible method, with the advantages of simple process flow, less one-time investment, low operating cost and wide applicability, and becomes a hot research topic in organic waste gas treatment technology. Vegetal oil, diesel oil, silicone oil and solar oil have been used to absorb benzene, toluene, xylene, methanol, etc ${ }^{[5-8]}$. But the absorption efficiency was greatly influenced by the contacting surface areas of streams to absorbents and the viscosities of absorbents.

The paper was focused on comparative investigation on the absorption performance of various composite absorbent for VOCs. The aim of this article is mainly to find relationship between interfacial tension and absorption efficiency.

\section{Experimental materials and methods}

Experimental Materials SDBS, analytical reagent, Tianjin Institute of Fine Chemical rehabilitation; Tween 20, chemically pure reagent, Tianjin Branch-Europe Chemical Reagent Co, Ltd; Toluene, butyl acetate, analytical reagent, Guangzhou Chemical Reagent Factory; Experimental water for the distilled water.

Experimental methods and procedures High-pressure drying air decompression is divided into two by the gas mass flow meter control flow, respectively, all the way into the gas containing toluene or butyl acetate liquid gas generating device, bring out organic vapor; and the other way gas in the buffer bottle confluence mix, then into the absorber and composite absorbent reverse absorption experiments. Absorption tower height of $650 \mathrm{~mm}$, diameter of $30 \mathrm{~mm}$, packing layer height of $400 \mathrm{~mm}$, 
ceramic ring packing $10 \mathrm{~mm} \times 10 \mathrm{~mm}$. Gas generator placed in a water bath at a constant temperature. By adjusting the gas flow to control the concentration of organic matter (about $500 \mathrm{mg} / \mathrm{m}^{3}$ ). Before and after the absorption of organic matter by the six-way valve quantitative, then determinated by Agilent $6890 \mathrm{~N}$ gas chromatograph hydrogen flame detector (FID).

\section{Experimental conclusions and discussion}

\section{Different composite absorbent critical micelle concentration}

As shown in Fig.1, the surface tension of the three composite absorbents rapidly decreases with increasing concentration at the initial low concentration, then slow down the rate of decline, until the maintenance of a relatively stable value, the corresponding surfactant concentration at the turning point of the surface tension curve from descending to slowing, that is critical micelle concentration, absorbent is also a more appropriate preparation of the concentration. Experiments show that the critical micelle concentration is the lowest Tween $20(80 \mathrm{mg} / \mathrm{L}), \operatorname{SCOP}(300 \mathrm{mg} / \mathrm{L})$ followed by SDBS $(420 \mathrm{mg} / \mathrm{L})$ the highest. The maximum surface tension reduction is SCOP, and its surface activity is also strongest.

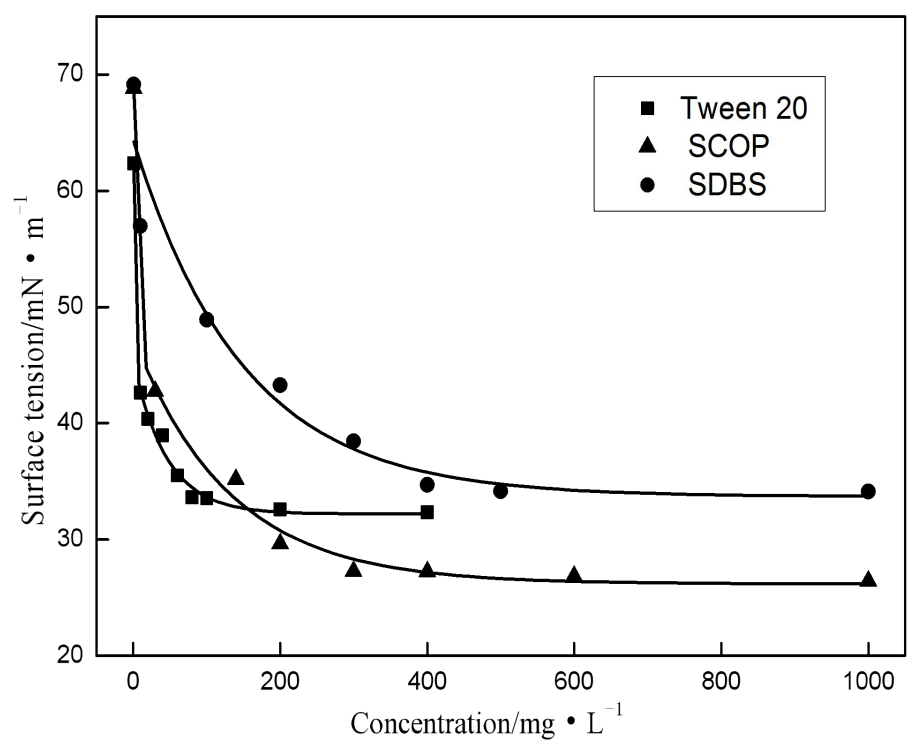

Fig. 1 three composite absorbent surface tension $\left(25^{\circ} \mathrm{C}\right)$

\section{Absorption of toluene/butyl acetate by different composite absorbent}

As shown in Table 1, the removal efficiencies of toluene, butyl acetate and the four absorbents are inversely proportional to the surface tension, and toluene is greater than butyl acetate. The performance of SCOP absorbent is the best. Tween 20 and SDBS have similar surface tension. However, the removal rate is quite different, the reason is that the molecular structure is different, SDBS is an ionic surfactant, in the absorption process its hydrophobic end is easy to adsorb on the absorbent surface to maintain low surface tension, thus maintaining a high removal rate. This also provides a basis for the screening of composite absorbent. 
Table 1 composite absorbent toluene, butyl acetate absorption effect

\begin{tabular}{|c|c|c|c|c|}
\hline \multirow{2}{*}{ Composite absorbent } & \multirow{2}{*}{$\begin{array}{l}\mathrm{CMC} \\
\mathrm{mg} / \mathrm{L}\end{array}$} & \multirow{2}{*}{$\begin{array}{c}\text { Surface Tension } \gamma \\
\mathrm{mN} / \mathrm{m}\end{array}$} & \multicolumn{2}{|c|}{ Average removal rate $(6 \mathrm{~h}) \%$} \\
\hline & & & Toluene & Butyl acetate \\
\hline SCOP & 300 & 27.26 & 91.26 & 83.28 \\
\hline SDBS & 420 & 34.35 & 62.39 & 57.32 \\
\hline Tween 20 & 80 & 33.60 & 36.56 & 34.45 \\
\hline $\mathrm{H}_{2} \mathrm{O}$ & l & 72.06 & 21.32 & 19.18 \\
\hline
\end{tabular}

\section{Surface tension between SCOP composite and toluene, butyl acetate}

As shown in Fig.2, as the SCOP concentration in the composite absorbent increases, the surface tension decreases, Toluene and butyl acetate removal rate increased rapidly, further verified the relationship between surface tension and removal rate. However, when the concentration reached CMC, the surface tension decreased slowly, the rate of removal also becomes slow. Although the concentration of the composite absorbent continues to increase little contribution to the removal rate, But in the experiment found that when the concentration exceeds CMC, As the composite absorbent formed micelles, increase the absorption capacity of pollutants, it is beneficial to maintain efficient absorption for a long time, absorption process changes from surface tension to surface tension and micellar solubilization.

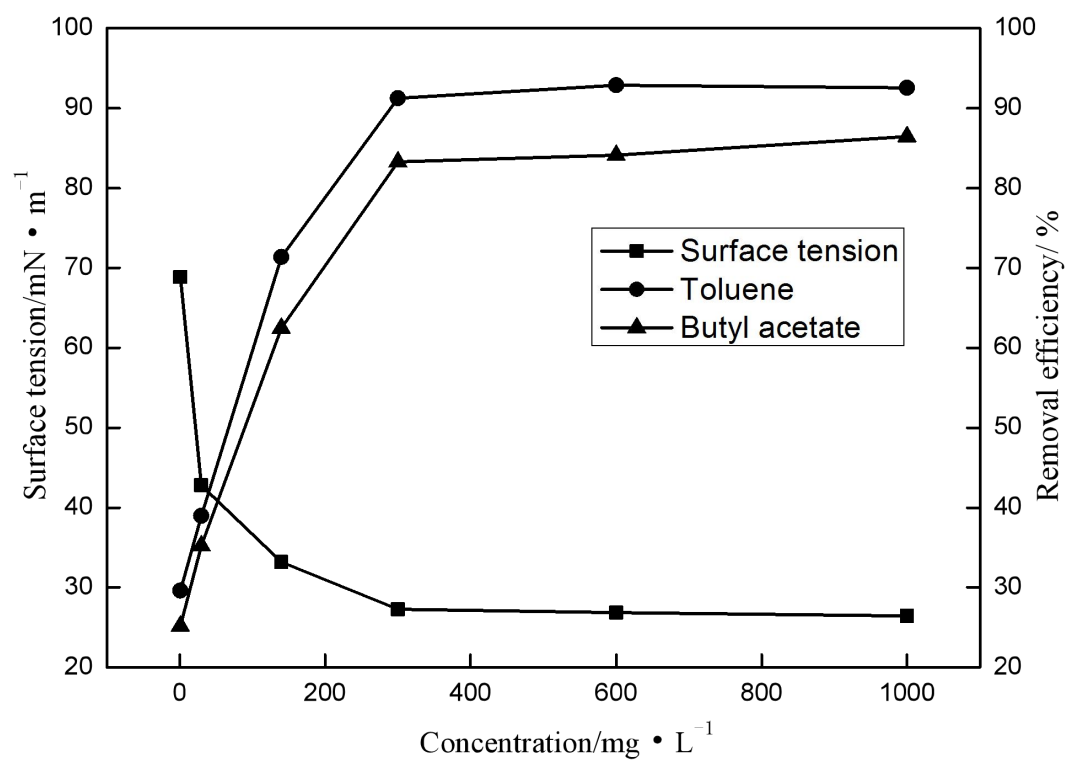

Fig 2 Surface tension of composite absorbent SCOP for toluene

According to the thermodynamic point of view, reduce the liquid surface area is to reduce the energy of the system process, in the absorption process organic pollutants from the gas phase into the liquid phase, Resulting in increased surface area, you need to work on it, work (A) size should be proportional to the new area $(\Delta \mathrm{S}):-\mathrm{A}=\gamma \Delta \mathrm{S}$. Absorption of a particular pollutant, $\Delta \mathrm{S}$ tends to a constant absorption depends on the surface tension $\gamma$. Therefore, the reduced surface tension of the composite absorbent facilitates the entry of contaminants from the gas phase to the liquid phase, thereby increasing the absorption efficiency. 


\section{Conclusions}

After adding SCOP in solvent water, absorbent surface can form a layer of adsorption layer, reduce surface tension and interfacial tension. Through the absorption efficiency and surface tension research results show that, SCOP absorbent the lowest surface tension, the best absorption. Surface tensions has an important influence on the absorption process, reduce the surface tension of the composite absorbent, is conducive to improving the removal of pollutants.

\section{Acknowledgements}

The research was supported by the Science and Technology Key Projects of Guangdong Province (2016B020241002, 2017A030223005), Nature Science Foundations of China (51408256), Special Funds for Research from the Environmental Charity Project of South China Institute of Environmental Sciences (PM-zx703-201701-011).

\section{References}

[1] Song Congbo, Wu Lin, Xie Yaochen, et al. Air pollution in China: Status and spatiotemporal variations [J]. Environmental Pollution, 2017, 227: 334-347.

[2] Shen Fuzhen, Ge Xinlei, Hu Jianlin, et al. Air pollution characteristics and health risks in Henan Province, China [J]. Environmental Research, 2017, 156: 625-634.

[3] Ban Jie, Zhou Lian, Zhang Yi. The health policy implications of individual adaptive behavior responses to smog pollution in urban China [J]. Environmental International, 2017, 106: 144 -152 .

[4] Sensor Geoffrey Gregis, Jean-Baptiste Sanchez, Igor Bezverkhyy, et al. colorimetric detector for lung cancer related volatile organic compounds based on cross-response mechanism[J]. Sensors and Actuators B , 2018, 256: 543-552

[5] T.K. Poddar, K.K. Sirkar, Henry's law constant for selected volatile organic compounds in high-boiling oils, J. Chem. Eng. Data, 1996(41):1329-1332.

[6] T.K. Poddar, S. Majumdar, K.K. Sirkar, Removal of VOCs from air by membranebased absorption and stripping, J. Membr. Sci. 1996 (120):221-237.

[7] N.I. Volodin, V.M. Puzyreva, V.E. Soroko, Absorption treatment of gases to remove impurities of organic solvents, Russ. J. Appl. Chem. 1997 (70) :1745-1747. 